\title{
Current approaches in retrieval and heart preservation
}

\author{
Maria Monteagudo Vela, Diana García Sáez, Andre R. Simon \\ Department of Cardiothoracic Transplantation and Mechanical Circulatory Support, Royal Brompton \& Harefield NHS Foundation Trust, \\ Harefield Hospital, London, UK \\ Correspondence to: Maria Monteagudo Vela. Department of Cardiothoracic Transplantation and Mechanical Circulatory Support, Royal Brompton \& \\ Harefield NHS Foundation Trust, Harefield Hospital, London, UK. Email: m.monteagudo-vela@rbht.nhs.uk.
}

\begin{abstract}
Fifty years after the first successful heart transplantation, despite multiple advances in the treatment of advanced acute and chronic heart failure, there is still no equivalent to heart transplantation as a long-term treatment for end-stage heart failure. Transplantation is, however, limited by the scarcity and quality of heart allografts. Donors are nowadays significantly older, particularly in European countries, and traumatic head injury as the cause of death has been replaced by intracerebral hemorrhage or hypoxic brain damage in the majority of cases. In addition, many donors have undergone extensive resuscitation efforts. Recipient characteristics have progressively changed too within the last couple of decades; recipients are older, often with comorbidities and nearly half of them are bridged to transplant with a wide variety of mechanical circulatory support devices. These developments have resulted in heart transplant surgery becoming significantly more challenging with longer more complex surgery and increased ischemia times for organs that were previously considered to be borderline or non-transplantable in many cases. To address this, several options have been explored within the last years and as a result, novel strategies have been developed and tested in order to optimize graft preservation and potentially increase the donor pool. The two notable developments are the ability to procure hearts from donors after circulatory death and the advent of ex-vivo perfusion of hearts. This technology has made the transplantation of extended criteria organs, including those from circulatory determined death (DCD) donors possible, and allow for out of body time of more than 12 hours in heart transplantation. In this review, we set out the basis of the current practices in organ procurement, and the opportunities for the future as demands for organ transplantation continue to increase.
\end{abstract}

Keywords: Heart retrieval; heart preservation; Organ Care System (OCS); circulatory determined death (DCD) donors

Submitted Sep 28, 2017. Accepted for publication Jan 06, 2018.

doi: 10.21037/acs.2018.01.06

View this article at: http://dx.doi.org/10.21037/acs.2018.01.06

\section{Introduction}

"I will give you a new heart and put a new spirit in you; I will remove from you your heart of stone and give you a beart of flesh." by Ezekiel 36:26.

In the last two decades, we have witnessed remarkable advances in the treatment of advanced, acute and chronic heart failure. Many new conservative/medical as well as surgical strategies have been implemented successfully and led to better quality of life as well as significant increases in the life span of these patients.
Ever-improving outcomes of long-term mechanical circulatory support devices provide a glimmer of hope for a real, epidemiologically relevant solution; however, heart transplantation still continues to be the gold standard and the most desired procedure in the treatment of end-stage heart failure today.

While many things have changed, the basic determinants of success in heart transplantation have not. They are in essence, the quality of the graft itself, the procurement, preservation and storage of the graft, including the ischemic time and the complexity of the operation and status of the 
recipient.

While we cannot influence the fact that our donors get older, have more co-morbidities and that organ quality declines as a result, nor the increase in complexity, age and inherent risk of our recipients, we can influence procurement and preservation, thus accessing previously non-accessible donor groups and counteracting the effects of certain parameters rendering the graft susceptible to injury and primary graft dysfunction (1).

In 1979, after successfully transplanting 19 hearts procured at distant hospitals with a mean ischemic time of 154 compared to 20 hearts procured locally with a mean ischemic time of 52 minutes without differences in mortality (32\% vs. $40 \%$ local), Stanford University Hospital concluded distant heart procurement could provide an expanded donor pool for potential cardiac recipients (2).

Approaches recently developed in order to enlarge the donor pool, include preservation and assessment of "extended criteria" donor allografts with ex-vivo normothermic perfusion machines and heart transplantation from donation after circulatory determined death (DCD) donors.

New technologies such as the OCS (Organ Care System, TransMedics) have clearly changed the scenario. The OCS is the first commercially available device that preserves the heart in a beating, perfused, normothermic and oxygenated state and allows the surgeons to assess transplantability of "extended criteria" donor hearts. Utilization of this technology has allowed for successful transplantation of hearts with significant left ventricular hypertrophy, reduced left ventricular ejection fraction (LVEF), palpable coronary artery disease or predicted long cold ischemic times after ex-situ assessment (3).

Another strategy that has increased the donor pool is the use of DCD hearts (4). These are hearts procured from patients with severe brain injury who do not meet the criteria of brain death and an infaust prognosis. Again, transplantation of these organs has been made possible by "selective resuscitation" of grafts after the cessation of heart beat utilizing perfusion technology; the first case of these being performed by the group at St. Vincent's Hospital, Sydney utilizing the OCS technology. Currently, only four hospitals worldwide utilize these types of donors with promising outcomes.

\section{Donor heart retrieval and myocardial protection}

The conclusive assessment about donor heart transplantability is made at the time of retrieval. Part of the standard evaluation and donor optimization in the UK includes performing a right heart catheterization via a Swan Ganz pulmonary artery catheter. Transoesophageal echocardiography at this stage can provide significant information about biventricular function and the presence of any ventricular hypertrophy or valvular abnormality. Coronary arteries are carefully inspected manually, especially in absence of a coronary angiogram, which is standard in most US donor organizations, but not available in the UK and many other countries.

The donor operation is usually performed as part of a multi-organ retrieval procedure. After the preparation of all organs is completed, the retrieval commences. At this point, the superior caval vein is ligated. The inferior caval vein is opened and the left atrium is vented, in order to prevent left ventricular distension. The ascending aorta is subsequently cross clamped and cold cardioplegia is administered via the aortic root to achieve hypothermic diastolic arrest. The heart is then quickly retrieved and classically packed in one or two litres of cardioplegia and placed in an ice box for cold storage.

Since the first heart transplant, different preservation solutions have been used for cold ischemic storage. Eurocollins (EC) was the most popular solution during the first year of the heart transplantation era, followed by University of Wisconsin (UW) solution. New solutions emerged as a popular alternative for heart transplantation; however, the comparison of randomized trials of histidinetryptophan-ketoglutarate (HTK), Celsior, and UW solutions has shown similar risk of delayed graft function after heart transplantation.

A review by Latchana et al. provides information on the biochemical comparison of common preservation solutions and relevant preservation studies (5).

\section{Cold ischemic storage}

Since the first successful human heart transplant was performed in 1967 , cold ischemic storage at $4{ }^{\circ} \mathrm{C}$ directly after procurement of the allograft until implantation in the recipient, has been the most widely used technique for preserving the retrieved heart. But while heart transplantation has become clinical routine on the basis of this technique, cold ischemic storage, has significant limitations. Prolonged ischemia time has been demonstrated to be one of the most important risk factors of early graft dysfunction of the implanted heart and 


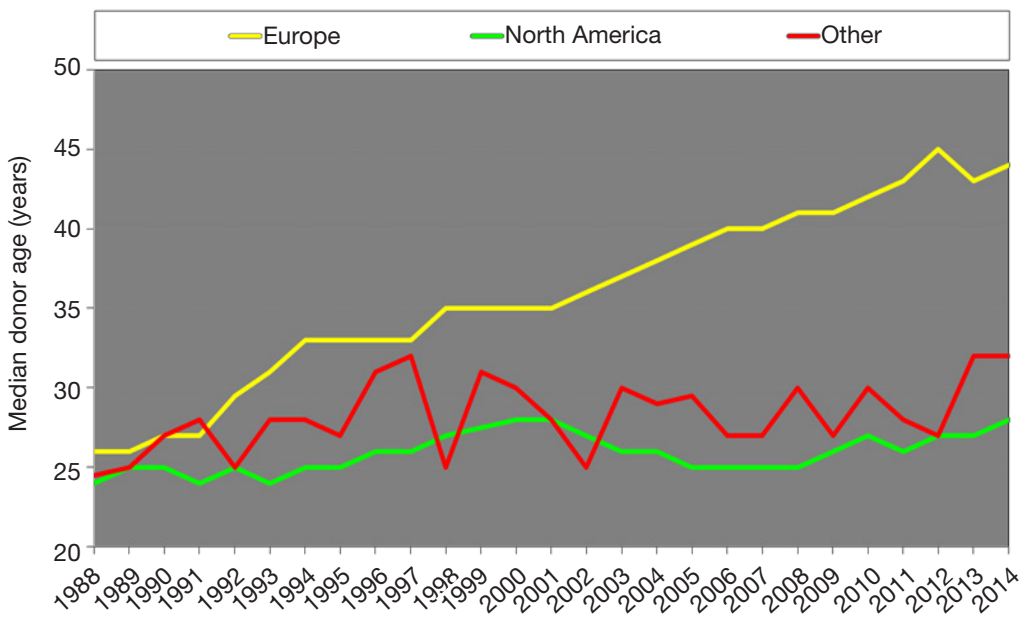

Figure 1 Median donor age worldwide.

therefore primary graft failure, the most frequent cause of death within the first month after heart transplantation. Every increase in this time means a higher possibility of developing early graft failure; however, as shown in both the ISHLT registry 2012, heart statics (6) and in the paper by Banner et al. (7), there is a significant and incremental increase of the risk of primary graft failure after 180 minutes of cold ischemic time. This has resulted in enormous organizational challenges, expedited surgery in which standard hemostasis and other steps have to be sacrificed in order to facilitate shorter ischemia times, the inability to transport organs across greater distances and the necessity to err on the side of caution if the risks of an extended ischemia seem significant, thus, effectively limiting the available donor pool even further.

Despite all efforts, and 20 years of investigations and different solutions, have not resulted in an ischemia time beyond a few hours (8).

There are, of course, alternatives in terms of heart protection and preservation. One promising method is transporting the heart in a beating normothermic perfused state. As explained further down, the proceed II trial shows the efficacy of transporting and preserving hearts with the OCS device. The short-term outcomes are similar when compared to cold ischemic storage, but show a significant reduction in the cold ischaemia time. This is a promising method that could increase the donor pool as well as provide a wide assessment of the heart.

Other possible strategies include CO-pretreatment (9) and remote preconditioning (10), but these remain experimental to this day.

\section{Current situation: donor-recipient combination} $(11,12)$

The many positive developments in the treatment of heart failure, have led to increasing demand of hearts and patients on the waiting list. This fact, added to the shortage of donors, creates ever increasing pressures on the waiting lists.

The ISHLT Heart Transplant Registry demonstrates that despite the increasing complexity of heart transplant recipients, results have improved progressively. Median recipient age has risen from under 40 to above 50 in the last three decades. Early survival rates tend to be lower in Europe and this may be related to the risk profile of the available cardiac donors; for example, the median donor age was around $25-30$ years old and is now near 45 years old, approaching 50 in the UK (Figure 1), as well as differing allocation strategies. In addition, the main cause of donor death has changed. Just a few decades ago, the majority of donors were young and the cause of death was traumatic head injury (Figure 2). Nowadays, because of improvements in road safety, seat belt use and other developments, we see older donors with hypoxic brain injury and additional comorbidities.

The recipient group has undergone significant changes too. Better conservative treatment, better outcomes in routine cardiac surgery and the introduction of ventricular assist devices into clinical routine have led to significant increase in the "diagnostic weight" of our patients. Today, approximately half of the patients receiving a heart transplant are on mechanical circulatory support and we find ourselves fighting with challenging surgeries with 


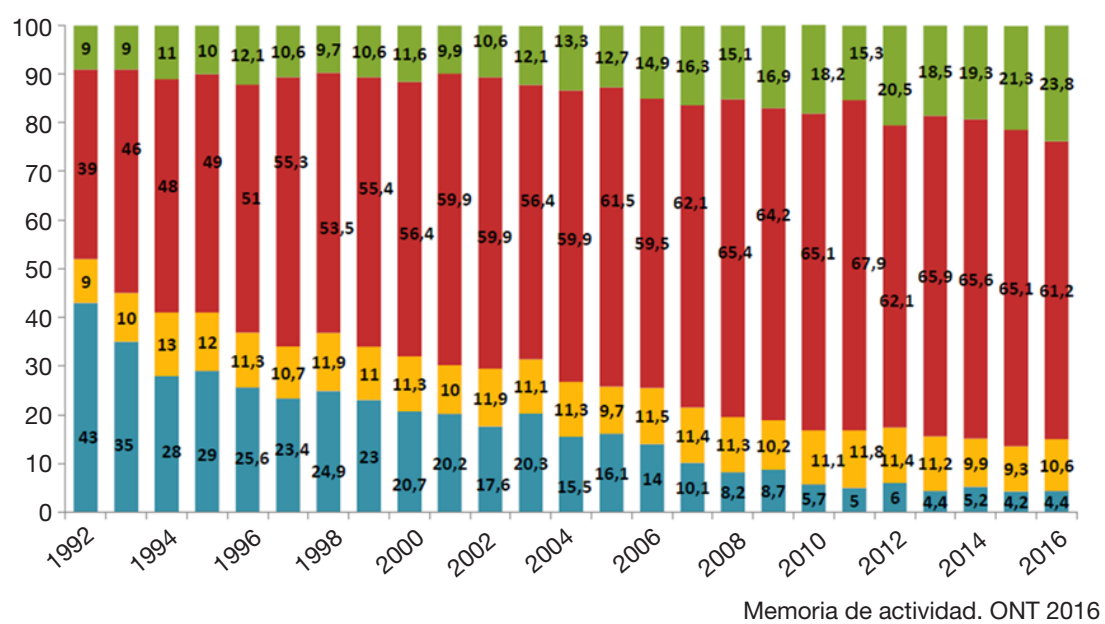

Figure 2 Donor cause of death. Blue: traffic trauma; yellow: non traffic trauma; red: stroke; green: other.

the donor heart in a box full of ice and the ischemia clock ticking; once more, increasing the recipient's risk.

\section{History of ex-vivo organ preservation $(13,14)$}

One hundred and fifty years ago, the idea to "restart" a "dead or dying heart" was utopia. The first isolated perfused heart was created by Elias Cyon, an assistant of Carl Ludwig in Leipzig at the time, in 1866. They resuscitated a frog heart, cannulating the aorta and the vena cava and perfusing it with rabbit serum. If they wanted the ventricle to generate pressure and eject the fluid, they needed to fill it with a certain amount of this serum.

Despite his great achievement, Cyon was an unfortunate and tragic character. As Kennan wrote: "His life remains shrouded in obscurity and can be reconstructed only uncertainly, from circumstantial rather than direct evidence. He never received recognition commensurate with his talents. He wrote in three modern languages. His views, especially with relation to philosophy and science, were ahead of his time."

Three decades later, Langendorff developed the first perfusion system for mammalian hearts. He demonstrated that perfusing the coronary arteries was enough to achieve the resuscitation of a dead heart, and that blood in the ventricles had no effect on excitability. In 1967, Neely and Morgan published the first "working" heart module in which the coronary perfusion was achieved via an ejecting ventricle into the aorta. The group of Pittsburgh developed in the 1980s a system of autoperfusion preservation. They removed canine and bovine heart-lung blocks and then preserved them with a system of normothermic coronary autoperfusion (15). In 1984, Wicomb and his group in South Africa published the first four cases in which they used a portable perfusion system. They retrieved hearts in the standard way but once excised, they perfused the hearts with a hypothermic solution after connecting the aorta to the system (16).

\section{Ex vivo normothermic preservation- TransMedics OCS}

There have been multiple attempts to create an ex-vivo perfusion platform to optimize organ preservation which could counteract the detrimental effect of prolonged cold ischemic storage. The TransMedics OCS is currently the only commercially available system that allows the donor heart to be preserved beating and perfused in normothermia until its implantation in the recipient. Because of this normothermic maintenance, the cold ischemic time is shortened, distant procurement of organs is achieved and the possibility of sharing organs across greater distances is now theoretically possible.

The OCS is a portable device with a wireless monitor, a perfusion module and a "maintenance" solution, that is given via a standard intravenous infusion pump into the donor blood circulating in the system (17). When utilized, 1,200-1,500 $\mathrm{mL}$ of donor blood is collected and used prior to cross-clamping to prime the perfusion module. The heart is retrieved in the standard way at the donor site, achieving cold cardioplegic arrest through the aortic root. After retrieving the heart and once in the back table the aorta and pulmonary artery are cannulated prior 
to ex vivo reperfusion. The heart is placed within the system and warm oxygenated donor blood is pumped into the aorta allowing the coronary arteries to be perfused. Both vena cava are closed and the coronary sinus flow then passes through the tricuspid valve and is ejected by the right ventricle into the pulmonary artery that is cannulated and returns the blood into a reservoir. Mean cold ischaemia time pre-OCS (from aortic cross-clamp to the initiation of the reperfusion in the system) is in our experience $23 \pm 6$ minutes, depending if only heart, or heart and lungs are retrieved, due longer time required for pneumoplegia delivery.

Grafts are usually perfused depending on the size, degree of ventricular hypertrophy and coronary artery disease, with an aortic flow of $1 \mathrm{~L} / \mathrm{min}$, aortic pressure around $70 \mathrm{mmHg}$, and a coronary blood flow around $700 \mathrm{~mL} / \mathrm{min}$. Arterial and venous lactate samples are regularly taken to assess the adequacy of perfusion and ensure a favorable myocardial lactate uptake. Synchronization of the pulsable perfusion pump to the electrocardiograph (ECG) during preservation allows lower aortic root pressure and coronary flow with optimized myocardial perfusion during ventricular diastole with the aim of reducing the risk of myocardial edema during prolonged preservation cases.

Grafts are perfused with warm, oxygenated donor blood supplemented with epinephrine infusion and a proprietary maintenance solution containing adenosine, glucose and several amino acids. Maintenance infusion requirements are titrated depending on aortic pressure which indicates coronary artery resistance to perfusion. As a standard, any significant increase in lactate within the perfusate above a threshold of $5 \mathrm{mmol} / \mathrm{L}$ prior to graft implantation is usually interpreted as a contraindication to implantation. Also, a higher lactate level in the venous efficient than the arterial side, is an indication of injury, damage or ischemia of the graft (18). However, this threshold somewhat depends on the donor lactate which could be in some cases much higher prior to blood drainage. A down-trending pattern with a favorable $\mathrm{A}-\mathrm{V}$ differential together with low requirement of adenosine infusion below $30-40 \mathrm{~mL} / \mathrm{h}$ are more relevant acceptance criteria in our experience $(19,20)$.

The PROCEED II (17) is the first multicenter, randomized non-inferiority trial that assessed the clinical outcomes of the OCS compared with the standard cold storage of donor hearts for transplantation. The trial showed no differences within the primary end point between patient and graft survival rates. Non-inferiority was shown with comparable outcomes between OCS and standard cold storage.

Mean total preservation time, defined as the time that the donor heart was out of the body, from the retrieval at the donor to the reperfusion in the recipient, was significantly longer in the OCS group than in the standard cold storage group. However, mean total cold ischaemia time was significantly shorter in the OCS group than in the standard cold storage group.

Within the last four years, more than 120 heart transplants have been performed at our institution following ex vivo normothermic preservation with the OCS in a combined setting of high-risk donors and recipients (19). Transplantation of hearts from donors outside of the standard criteria has been found to be safe and feasible even in a high-risk recipient setting with at least forty percent of them on mechanical circulatory support. We understand as outside of the standard criteria; left ventricular dysfunction (LVEF $<45 \%$ ), prolonged donor cardiac arrest, of unknown duration in some cases, with a median time of 30 minutes, left ventricular hypertrophy defined as interventricular septal thickness of 15-19 $\mathrm{mm}$ in diastole. We have also transplanted 10 grafts with coronary artery disease, in absence of donor coronary angiography, which was either palpable and deemed not significant during inspection, or not palpable but suspected during ex-situ preservation, and later confirmed post-transplant. Coronary artery disease can be suspected with persistent high aortic pressures, not responsive to increased adenosine infusion and/or an increase or elevation of lactate.

As the ISHLT registry continues to identify LVAD bridging as a risk factor for increased mortality after transplantation, ex-vivo normothermic preservation may play an important role in logistics and surgical strategy. It allows meticulous preparation of the recipients with LVADs and removes the element of time pressure from the equation.

\section{Donation after circulatory death}

International registries show a trend towards heart transplantation donors increasing in age and presenting with more co-morbidities, thus the transplanted organs of today would have been previously classified as marginal. This shift in acceptance criteria, necessary to counteract the changes in the donor pool is limited by current assessment, procurement and preservation technique. Thus, the pool of "good" quality hearts available for transplantation shrinks.

Our alternative group of donors would be the DCD pool; indeed, these are often younger and come with less 


\begin{tabular}{llll}
\multicolumn{2}{l}{ Table 1 Maastricht categories of DCD } & Locations of practiced \\
\hline Category & Description & Type of DCD & ED in a transplant centre \\
\hline I & Dead on arrival & Uncontrolled & ED in a transplant centre \\
II & Unsuccessful resuscitation & Uncontrolled & ICU and ED \\
III & Anticipated cardiac arrest & Controlled & ICU and ED \\
IV & Cardiac arrest in a brain-dead donor & Controlled & ICU in a transplant centre \\
V & Unexpected arrest in ICU patient & Uncontrolled &
\end{tabular}

co-morbidities. But while transplantation of organs from donors after circulatory death has been a clinical reality in other areas, heart transplantation has not been possible until recently (21). DCD heart transplantation may sound like science fiction, but it is not a new concept. In 1967, prior to the introduction of brain death legislation, a heart procured after circulatory death allowed Christiaan Barnard to perform the first heart transplant in Cape Town (South Africa). In this first transplant, the donor and the recipient were in adjacent operating theatres in order to harmonize recipient and donor surgery to keep the ischemic time to an absolute minimum (22).

Survival rates after these first heart transplants, however, were very low and patients died of postoperative complications, allograft rejection and opportunistic infections (23). Since then, while surgical technique has remained largely unchanged, the introduction of cyclosporine into immunosuppressive therapy, serial biopsies to assess the rejection grade, cold preservation of the allograft, prophylaxis of infections, additional medication such as statins, and cold ischemic shortage have turned heart transplant into a clinical success story.

Donation after brain death (DBD) donation radically changed the process of retrieval with the introduction of brain death legislation. Donation after circulatory death came to a halt, allowing for procurement after a controlled cardioplegic arrest, preserving the heart with a standard cold storage method and avoiding the need of transferring the donors to the recipients' hospital. In addition, cold storage permitted the distant procurement of organs which was inconceivable before.

In 1995, the Maastricht categories regarding DCD were formulated (Table 1). Today, DCD donors are category III, meaning a controlled withdrawal of life support in a patient that has suffered irreversible and extensive brain injury but does not meet brain death criteria. There should be consent between the family of the patient and the intensive care unit (ICU) physicians.

The successful introduction of normothermic, portable, ex situ perfusion gave rise to the idea to utilize this concept in a clinical DCD heart procurement setting. After simulation in animal experiments, it was put into clinical practice by the team at St. Vincent's Hospital, Sydney, initially reporting a case series of three patients (24). Afterwards, both Papworth and Harefield in the UK added their names to the list of pioneers performing these transplants. Currently, more than 50 DCD hearts have been transplanted within four hospitals worldwide (Sydney, Australia; Papworth, UK; Harefield, UK; Manchester, UK) with outcomes easily comparable to those from donors after brain death.

As the main concern of DCD hearts is in the possible myocardial injury due to the warm ischemic time from withdrawal of life sustaining therapies until the heart is either perfused in situ or until cardioplegia is delivered and its effect on function, a functional assessment between death and cessation of heart beat and acceptance is vital.

In order to achieve this, two concepts of reperfusing a DCD heart prior to transplantation are utilized; one technique entails in-situ normothermic regional perfusion (NRP) of the donor following circulatory death, who is placed in central extracorporeal life support (3). For that purpose, cannulae are inserted into the ascending aorta and right atrium with cerebral exclusion by clamping neck vessels, before restoring perfusion. Once the heart is ejecting, the donor is weaned from NRP support and graft function is assessed with transoesophageal echocardiogram and cardiac output monitoring with a Swan Ganz catheter commencing retrieval, the graft is transported on the OCS in order to avoid the additional result of prolonged cold ischemic storage. This 
technique has been developed by Papworth Hospital and is applied to a few of their zonal referral donor centers. It has also generated significant ethical discussions in the transplant community mainly regarding re-establishing circulation in a donor following circulatory death, and regarding the remote possibility of brain reperfusion via the vertebral arteries in case of the left subclavian artery not being clamped properly.

The other, most widely used technique for DCD heart retrieval is the direct procurement of the graft after circulatory death and subsequent $e x$-situ reperfusion with OCS, originally described by the Sydney group. This obviates the extra costs, logistical problems and ethical discussions pertaining to the in situ NRP of donor hearts. Direct retrieval is the standard approach used at our institution and Manchester. There are minor variations across the four centers using this technique mainly regarding donor blood drainage technique (25).

To this day, more than 50 heart transplant procedures have been performed worldwide with very positive outcomes. However, some centers remain reluctant to use these types of donor. Future challenges will include expanding the acceptance criteria of donor age and warm ischemic times, as well as monitoring the long-term outcomes of DCD heart transplantation.

\section{Future}

The future is, as always, uncertain. What is clear however, is that in 20 years, only minor changes have occurred in heart retrieval and preservation techniques and that these could counteract the changes in recipients and the donor pool only partially. Devices for normothermic ex-vivo perfusion like the OCS and the "normalization" of using hearts from DCD donors have changed this situation dramatically and clearly contributed to an increase of the donor pool as well as improved preservation of organs.

Further research is required in this field as a shortage of organs remains a reality and investment will be needed in the development of new preservation strategies. In an effort to increase heart preservation duration, this could include the usage of hibernation trigger factors which could be administered to donors before organ harvest. Another idea includes sub-zero non-freezing preservation (26) using a variable magnetic field which has been tested in a porcine model, which has resulted in a significant suppression of anaerobic metabolism and myocardial protection.

Lastly cryopreservation may enable long term storage of grafts. Organ perfusion and preservation is developing and it is likely that we will see further developments of the technology. It is, however, high cost medicine and in an age of rationalization of resources, it will have to pass the test of cost effectiveness in order to be widely accepted and used.

Nevertheless, with all these developments, heart transplantation seems here to remain and may have an exciting future.

\section{Conclusions}

Heart transplantation continues to be the gold standard treatment of end-stage heart failure. Donors, as well as recipients, will continue to change in terms of age and comorbidities. The great advance in mechanical circulatory support devices has not yet resulted in a true alternative to heart transplant. As a result, it is filling the waiting list with complex patients that will need challenging surgeries during heart transplantation. Thus, the conundrum has not changed. We need more organs and we need to improve preservation. It is well known that prolonged graft ischemic time is a significant risk factor for primary graft dysfunction and therefore for early graft failure, the most frequent cause of death in the first 30 days after heart transplantation. Normothermic ex-vivo preservation with the OCS, significantly reduces the cold ischaemia time and allows distant procurement and biochemical and functional assessment of the donor heart. DCD heart transplantation demonstrates positive outcomes. Both strategies are necessary in an era where organ shortage and increasing waiting lists are a reality. The results are quite exciting in how this new technology is making a change in heart transplantation. Further applications of all these advances will be crucial for ex-vivo perfusion, marginal donors' resuscitation and regeneration of myocardial tissue.

\section{Acknowledgements}

None.

\section{Footnote}

Conflicts of Interest: The authors have no conflicts of interest to declare.

\section{References}

1. Messer S, Ardehali A, Tsui S. Normotemic donor heart perfusion, current clinical experience and the future. 
Transpl Int 2015;28:634-42.

2. Watson DC, Reitz BA, Baumgartner WA, et al.

Distant heart procurement for transplantation. Surgery 1979;86:56-9.

3. García Sáez D, Zych B, Mohite P, et al. Midterm Follow up of Heart Transplantation with Routine Ex-Vivo Normothermic Preservation with Adverse Donor - Recipient Risk Profile. J Heart Lung Transplant 2017;36:S17.

4. Messer SJ, Axell RG, Colah S, et al. Functional assessment and transplantation of the donor heart after circulatory death. J Heart Lung Transplant 2016;35:1443-52.

5. Latchana N, Peck JR, Whitson B, et al. Preservation solutions for cardiac and pulmonary donor grafts, a review of the current literature. J Thorac Dis 2014;6:1143-9.

6. Heart Statistics. ISHLT Registry 2012. Available online: https://www.ishlt.org/registries/slides.asp?slides=heartLun gRegistry\&year $=2012$

7. Banner NR, Thomas HL, Curnow E, et al. The importance of cold and warm cardiac ischemia for survival after heart transplantation. Transplantation 2008;86:542-7.

8. Heart Statistics. ISHLT Registry 2016. Available online: https://www.ishlt.org/registries/slides.asp?slides=heartLun gRegistry\&year $=2016$

9. Fujisaki N, Kohama K, Nishimura T, et al. Donor pretreatment with carbon monoxide prevents ischemia reperfusion injury following heart transplantation in rats. Med Gas Res 2016;6:122-9.

10. Hausenloy DJ, Candilio L, Evans R, et al. ERICCA Trial Investigators. Remote Ischemic Preconditioning and Outcomes of Cardiac Surgery. N Engl J Med 2015;373:1408-17.

11. Heart Overall Statistics. ISHLT Registry 2016. Available online: https://www.ishlt.org/registries/slides.asp? slides=he artLungRegistry\&year $=2016$

12. Memoria de Actividad. ONT 2016. Available online: http://www.ont.es/infesp/Memorias/Memoria\%20 Introducci\% C3\%B3n.pdf

13. Zimmer HG. The Isolated Perfused Heart and Its Pioneers. News Physiol Sci 1998;13:203-10.

14. Zimmer HG. Profiles in Cardiology. Ilya Fadeyevich Tsion, alias Elias Cyon, alias Élie de Cyon. Clin Cardiol 2004;27:584-5.

15. Ladowski JS, Kapelanski DP, Teodori MF, et al. Use of

Cite this article as: Monteagudo Vela M, García Sáez D, Simon AR. Current approaches in retrieval and heart preservation. Ann Cardiothorac Surg 2018;7(1):67-74. doi: 10.21037/acs.2018.01.06 autoperfusion for distant procurement of heart-lung allografts. J Heart Transplant 1985;4:330-3.

16. Wicomb WN, Cooper DK, Novitzky D, et al. Cardiac transplantation following storage of the donor heart by a portable hypothermic perfusion system. Ann Thorac Surg 1984;37:243-8.

17. Ardehali A, Esmailian F, Deng M, et al. Ex-vivo perfusion of donor hearts for human heart transplantation (PROCEED II) a prospective, open-label, multicentre, randomised non-inferiority trial. Lancet 2015;385:2577-84.

18. Hamed A, Tsui S, Huberm J, et al. Serum lactate is a highly sensitive and specific predictor of post-cardiac transplant outcomes using The Organ Care System. J Heart Lung Transplant 2009;28:S71.

19. García Sáez D, Zych B, Sabashnikov A, et al. Evaluation of the Organ Care System in heart transplantation with an adverse donor recipient profile. Ann Thorac Surg 2014;98:2099-105; discussion 2105-6.

20. Dhital K, Simon A, Macdonald P. Ex-vivo Donor Heart Perfusion, Testing the Limits of Cardiac Resilience. Heart Lung Circ 2015;24:1191-2.

21. Dhital KK, Chew HC, Macdonald PS. Donation after circulatory death heart transplantation. Curr Opin Organ Transplant 2017;22:189-97.

22. Barnard CN. The operation. A human cardiac transplant: an interim report of a successful operation performed at Groote Schuur Hospital, Cape Town. S Afr Med J 1967;41:1271-4.

23. Dhital KK, Iyer A, Connellan M, et al. Adult heart transplantation with distant procurement and ex vivo preservation of donor hearts after circulatory death, a case series. Lancet 2015;385:2585-91.

24. Hunt SA. Taking heart, cardiac transplantation past, present, and future. N Engl J Med 2006;355:231-5.

25. García Sáez D, Bowles CT, Mohite PN, et al. Heart transplantation after donor circulatory death in patients bridged to transplant with implantable left ventricular assist devices. J Heart Lung Transplant 2016;35:1255-60.

26. Bruinsma BG, Uygun K. Subzero organ preservation: the dawn of a new ice age? Curr Opin Organ Transplant 2017;22:281-6. 